\title{
One-dimensional Fe surface states confined by self-assembled carbon chains at the $\mathrm{Fe}(001)$ surface
}

\author{
G. Trimarchi and N. Binggeli \\ INFM DEMOCRITOS National Simulation Center and The \\ Abdus Salam International Center for Theoretical Physics, \\ Strada Costiera 11, 34014 Trieste, Italy
}

(Dated: November 21, 2018)

\begin{abstract}
A $\mathrm{c}(3 \sqrt{2} \times \sqrt{2})$ reconstructed structure of the $\mathrm{Fe}(001)$ surface with self-assembled $\mathrm{C}$ zigzag chains has been recently observed experimentally. Using ab initio density-functional calculations, we address the effect of the $\mathrm{C}$ zigzag chains on the electronic structure of this surface. We find that the formation of the $\mathrm{C}$ chains produces one-dimensional surface states localized along the zigzag chains. These states are spin-polarized and derive from preexisting two-dimensional Fe $d_{3 z^{2}-r^{2}}$-like surface states of the clean Fe(001) surface. The simulation of the STM image allows us to assign the chain-like structure, observed in STM experiments, to the one-dimensional Fe surface states laterally confined within the $\mathrm{C}$ zigzag stripes.
\end{abstract}

PACS numbers: 73.20.At, 68.37.Ef, 68.35.Dv 
The confinement of metal surface states using lateral nano or atomic-scale structures has attracted much interest in recent years. Well-known examples are "quantum corrals", where free-electron-like standing waves of a metal surface are confined within corral-like rings composed of ad-atoms. $\stackrel{1}{-}$ Other striking examples include confined surface states at vicinal or decorated stepped surfaces ${ }^{2}$ and one-dimensional surface states at domain boundaries in two-dimensional alloys. ${ }^{3}$ The study of metal surface states and their manipulation via lateral structures is not only of fundamental interest, i.e., to investigate quantum interferences and/or low-dimensional systems, but also of practical importance, since surface states are known to influence the adsorption characteristics of surfaces and, in the case of transitionmetal surfaces, also the surface magnetism. ${ }^{\underline{4}}$

Self-assembling has been the focus of extensive investigations lately, as a promising tool to build ordered nano or atomic-scale structures, without the direct manipulation of atoms or molecules. This approach has been used, e.g., to grow semiconductor quantum $\operatorname{dots}^{5}$ and semiconductor and metal quantum wires $\underline{\underline{6}}$ on semiconductor surfaces. In general, in self-assembled systems, the confinement concerns the electronic states of the self-organized species. The possibility, however, to manipulate surface states of the substrate, and in particular to laterally confine them within regular patterns via self-organized structures, is also of special interest as a potential approach to engineer metal surface properties.

Very recently, an interesting case of self-organized chains of non-magnetic impurities on a magnetic metal was reported. A Borges-garden-like arrangement of C stripes on Fe(001), corresponding to a $\mathrm{C}$ coverage of $2 / 3$ monolayer (ML), was induced via surface segregation of bulk C impurities. ${ }^{7.8}$ Scanning tunneling microscopy (STM) indicated the presence of self-assembled C zigzag chains forming a regular pattern over a nanometer lateral scale, corresponding to a $c(3 \sqrt{2} \times \sqrt{2})$ reconstructed structure.

In the present work, using state-of-the-art ab initio calculations, we examine this $c(3 \sqrt{2} \times$ $\sqrt{2}$ ) reconstructed structure, and show that the formation of the $\mathrm{C}$ chains gives rise to onedimensional Fe surface states close to the Fermi energy. These states are spin-polarized and laterally confined within the $\mathrm{C}$ zigzag stripes. We identify the origin of their confinement, and show that such surface states are responsible for the chain-like structures observed in STM experiments. We also predict that the one-dimensional Fe states give rise to a significant feature in the local density of states, which should be measurable by scanning tunneling spectroscopy. The surface states we predict exhibit some similarities with the localized Fe 


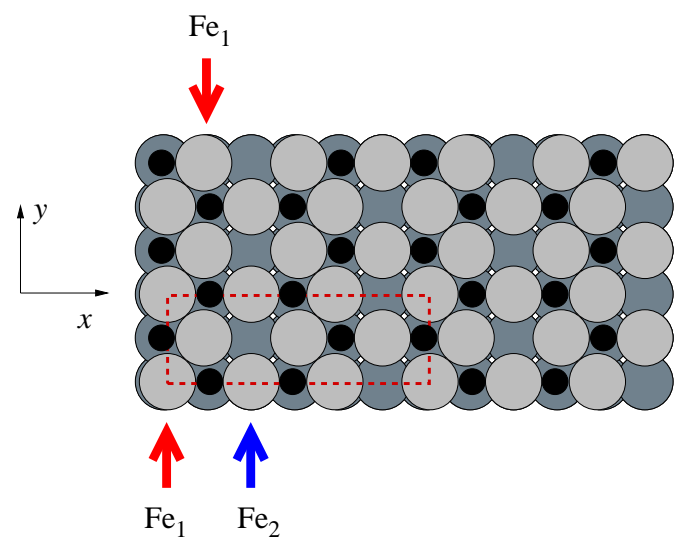

FIG. 1: (Color online) Top view of the $\mathrm{C} / \mathrm{Fe}(001) \mathrm{c}(3 \sqrt{2} \times \sqrt{2})$ surface. The dashed box indicates the $\mathrm{c}(3 \sqrt{2} \times \sqrt{2})$ surface unit cell. Small (large) disks corresponds to $\mathrm{C}$ (Fe) atoms; light (dark) gray disks indicate top (second layer) Fe atoms. The carbon atoms sit on hollow sites and lie $\sim 0.2$ $\AA$ above the outermost $\mathrm{Fe}$ atoms. $\mathrm{Fe}_{1(2)}$ indicates $\mathrm{Fe}$ surface atoms enclosed within (in between) the $\mathrm{C}$ stripes.

states found in Ref. 3 at Fe-rich domain boundaries on the $\mathrm{Si} / \mathrm{Fe}(001)$ surface alloy with $\sim 35 \% \mathrm{Si}$. In the latter alloy system, however, the observed chains of Fe surface states are disordered, and originate from a different local atomic geometry, i.e., from Si occupying substitutional sites within the $\mathrm{Fe}(001)$ surface layer, whereas the $\mathrm{C}$ atoms segregate at hollow sites and produce self-organized stripes.

Our first-principle calculations are performed within density functional theory, using the spin-polarized Perdew-Burke-Ernzerhof $f^{9}$ exchange-correlation functional and Vanderbilt ${ }^{10}$ ultra-soft pseudopotentials with a plane-wane basis set. The kinetic-energy cutoffs for the wave functions and electronic density are set to 27 Ry and 270 Ry, respectively. The sampling of the Brillouin Zone (BZ) is done with a Monkhorst-Pack (MP) grid, and we use a Gaussian smearing of the electronic levels with a full-width at half maximum of 0.02 Ry to determine the Fermi energy. The theoretical lattice constant $a$ of bulk iron is $2.86 \AA$ (compared to the experimental value of $2.87 \AA)$. To model the $\mathrm{C} / \mathrm{Fe}(001) \mathrm{c}(3 \sqrt{2} \times \sqrt{2})$ surface, we use a symmetric slab of $9 \mathrm{Fe}$ atomic layers with $4 \mathrm{C}$ atoms per $\mathrm{c}(3 \sqrt{2} \times \sqrt{2})$ surface unit. The thickness of the vacuum regions separating the periodic images of the slab corresponds to 9 $\mathrm{Fe}(001)$ atomic layers (i.e., $\sim 12.85 \AA$ ) and we use a $(2,6,1) \mathrm{MP}$ grid for the BZ integrations. In Fig. 1, we display the equilibrium atomic structure of the $\mathrm{C} / \mathrm{Fe}(001)$ with $2 / 3 \mathrm{ML}$ of 
C. The zigzag-chain structure is the lowest-energy configuration we obtain for this coverage and reconstruction. The details of the structural investigation will be presented elsewhere, $\stackrel{8}{-}$ it is only important here to note that the $\mathrm{C}$ atoms of the zigzag chains occupy hollow sites and display a strong relaxation towards the Fe surface layer, i.e, their equilibrium position is only $0.2 \AA$ higher than that of the outermost Fe atoms.

In connection with the interpretation of the STM experiments, we have examined the spatial behavior in the vacuum, near the surface, of the local density of empty electronic states integrated between the Fermi energy, $E_{\mathrm{F}}$, and $E_{\mathrm{F}}+\Delta \varepsilon$, with $\Delta \varepsilon$ taken as large as $1 \mathrm{eV}$ and as low as $0.2 \mathrm{eV}$. In Fig. 20 (2) $I(\Delta \varepsilon, \boldsymbol{r})$, obtained for $\Delta \varepsilon=+0.4 \mathrm{eV}(+0.9 \mathrm{eV})$, in a (001) plane located in the vacuum at a distance $d \approx 2.6 \AA$ from the outermost $\mathrm{C}$ layer. Within the Tersoff-Hamann approximation, $I\left(\Delta \varepsilon, d, \boldsymbol{r}_{\|}\right)$is proportional to the tunneling current corresponding to a bias $\Delta \varepsilon$, tip distance

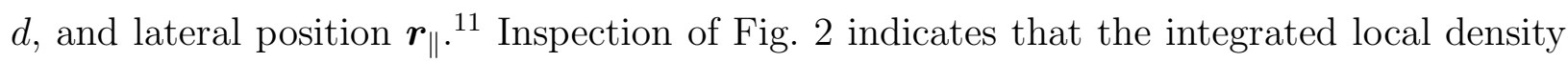
of states has its maxima positioned on top of the Fe surface atoms enclosed within the $\mathrm{C}$ stripes $\left(\mathrm{Fe}_{1}\right.$ atoms in Fig. (1). This gives rise to a zigzag chain structure in the simulated STM image at positive bias (see Fig. 2r), in good agreement with the experimental STM images,,$\frac{7.8}{.}$ even though, counter-intuitively, bright spots do not correspond to the $\mathrm{C}$ atoms of the zigzag chains, but to the Fe atoms enclosed within their stripes. We note that for all $\Delta \varepsilon$ examined, from 0.2 to $1 \mathrm{eV}$, and for all distances $d$ considered, from 2.6 to $3.2 \AA$, the integrated local density of states exhibits a qualitatively similar behavior, i.e., a zigzag chain arrangement of maxima (bright spots) located on the $\mathrm{Fe}_{1}$ atoms.

To better understand this behavior, we have investigated the energy distribution and the $k$-space dispersion of the tunneling states. In Fig. 3, we display the local density of states (LDOS) integrated in the vacuum region between $d=3.2 \AA$ and the middle of the vacuum slab $(d \approx 6.7 \AA)$. The separate contributions from areas enclosed within and in-between the carbon stripes (with area ratio 2:1) are also shown, as well as the specific contributions from the minority-spin states enclosed within these regions. The minority-spin states are responsible for a broad main feature, located at $\sim 0.6 \mathrm{eV}$ above $E_{\mathrm{F}}$, and a smaller feature, at $\sim 0.8 \mathrm{eV}$. The former (latter) is related mainly to states enclosed within (in-between) the $\mathrm{C}$ stripes. We note that the majority-spin states do not give rise to any significant feature up to $\sim 2.0 \mathrm{eV}$ above $E_{\mathrm{F}}$.

In Fig. [4 we display the band dispersion of the minority-spin tunneling states with the 

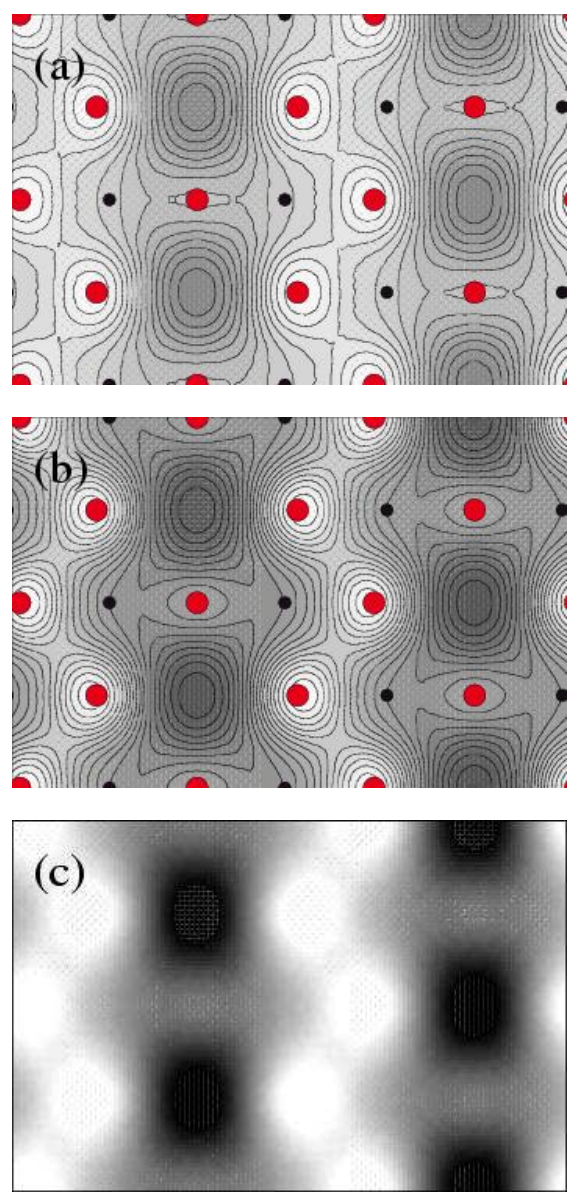

FIG. 2: (Color online) Contour plots in a (001) plane, at a distance $\mathrm{d} \approx 2.6 \AA$ from the carbon surface layer, of the local density of states integrated over the energy window $\left[E_{F}, E_{F}+\Delta \varepsilon\right]$, where $\mathrm{E}_{F}$ is the Fermi energy and $\Delta \varepsilon=0.4$ (a) and $0.9(\mathrm{~b}) \mathrm{eV}$. The positions of the Fe atoms (large disks) and $\mathrm{C}$ atoms (small disks) are also indicated. The spacing between the contours in (a) and (b) is respectively $0.78 \times 10^{-6} \mathrm{e} / \mathrm{bohr}^{3}$ and $3.2 \times 10^{-6} \mathrm{e} / \mathrm{bohr}^{3}$, with the contour of lowest density corresponding respectively to $5.9 \times 10^{-6}$ and $4.9 \times 10^{-5} \mathrm{e} / \mathrm{bohr}^{3}$. Panel (c) shows a gray scale plot of the integrated local density of states obtained for $\Delta \varepsilon=0.4 \mathrm{eV}$ in a $(001)$ plane at $\mathrm{d} \approx 3.0 \AA$ : light/dark gray corresponds to large/small values of the integrated local density of states.

largest integrated probability density in the vacuum beyond $d=2.3 \AA$. The band dispersion is shown along the $\bar{\Gamma}-\bar{K}_{\|}^{B Z}$ line parallel to the $\mathrm{C}$ chains in the 2-dimensional BZ of the $c(3 \sqrt{2} \times \sqrt{2})$ surface; $\bar{K}_{\|}^{B Z}$ stands for the zone-edge $k$ point $\left(\frac{\pi}{2 a}, \frac{\pi}{2 a}\right)$. Above the Fermi energy, we find three bands of tunneling states which yield the main contribution to the LDOS features observed between $0.3 \mathrm{eV}$ and $1.0 \mathrm{eV}$ in Fig. 3. The lowest-energy feature 
is associated with the two bands between $0.3 \mathrm{eV}$ and $0.7 \mathrm{eV}$ and the higher-energy feature with the band at $\sim 0.8 \mathrm{eV}$. These three bands derive from minority-spin surface states of the clean $\mathrm{Fe}(001)$ surface, which persist at the $2 / 3 \mathrm{C}$ covered surface. The probability densities of the corresponding three surface-band states at $\bar{\Gamma}$ are shown in Fig. [5 (a), (b), and (c), in a (110) atomic plane perpendicular to the $\mathrm{C}$ chains. The two lowest-energy states include mainly $d_{3 z^{2}-r^{2}}$ like orbitals of the $\mathrm{Fe}_{1}$ atoms, whereas the higher-energy state involves predominantly $d_{3 z^{2}-r^{2}}$ like orbitals of the $\mathrm{Fe}_{2}$ atoms. We note that these states are also mostly localized at the surface, as more than $60 \%$ of their electronic charge is located on the two outermost Fe atomic layers.

The minority-spin $d_{3 z^{2}-r^{2}}$ like surface states of the Fe(001) surface have been shown to induce a peak at about $0.2 \mathrm{eV}$ above $E_{\mathrm{F}}$ in scanning tunneling spectroscopy (STS) of the clean $\mathrm{Fe}(001)$ surface. ${ }^{3.12}$ In the presence of segregated $\mathrm{C}$, we find that such states are shifted to higher energy, giving rise to the two features, between 0.3 and $1 \mathrm{eV}$, in the LDOS in Fig. 3. We note that similar shifts to higher energy of reminiscent surface states of the transition-metal substrate in the presence of segregated impurities have been observed at domain boundaries on the $\mathrm{Si} / \mathrm{Fe}(001)$ surface alloy ${ }^{3}$ and at contaminated C-rich areas on the $\mathrm{V}(001)$ surface $^{13}$ In our case, however, we can distinguish two features: a low-energy feature, corresponding to minority-spin Fe $d_{3 z^{2}-r^{2}}$ like surface states localized within the $\mathrm{C}$ stripes, and a higher-energy feature, related mainly to $\mathrm{Fe} d_{3 z^{2}-r^{2}}$ like states located in between the $\mathrm{C}$ stripes. The former states explain the zigzag chain structure observed in the simulated STM image (Fig. 2r), while the latter are responsible for some additional weak features, located on the $\mathrm{Fe}_{2}$ atoms, in the LDOS with increased $\Delta \varepsilon$ (Fig. $2 \mathrm{~b}$ ).

Both the global shift to higher energy of the tunneling Fe surface states and their splitting into states located either within or in between the $\mathrm{C}$ stripes can be explained considering the changes in the electrostatic potential induced by the Carbon atoms at the surface. The energy shift is attributed to the change in the work function and the splitting to the induced corrugation of the electrostatic potential. The calculated work function of the Fe(001) surface changes from $4.0 \mathrm{eV}$, for the clean surface, to $4.8 \mathrm{eV}$, for the $2 / 3 \mathrm{C}$ covered $c(3 \sqrt{2} \times \sqrt{2})$ surface. This corresponds to a $0.8 \mathrm{eV}$ increase in the electrostatic potential step at the surface in the presence of the carbon. This increase in the potential step occurs between $d \approx 0$ and $d \approx a$ (see Fig. 5 $\mathrm{d}$ ), and thus shifts surface states which extend far into the vacuum, and in particular the tunneling states of Fig. 4, to higher energy with respect to 


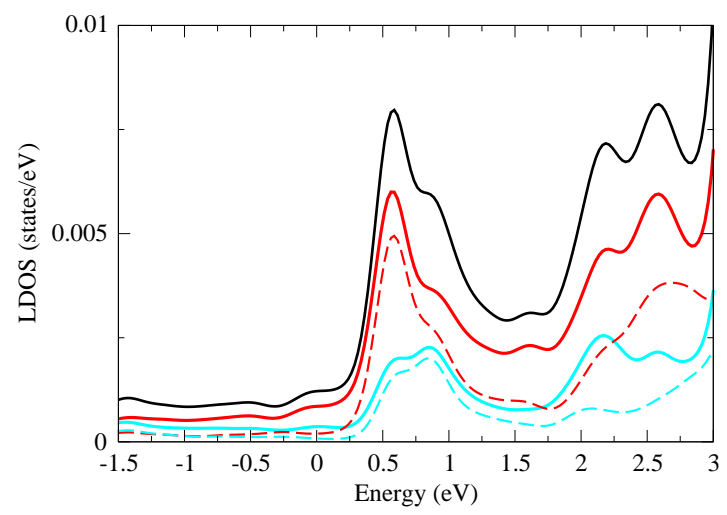

FIG. 3: (Color online) Local Density of States integrated over the vacuum space between a distance $\mathrm{d}=3.2 \AA$, measured from the outermost $\mathrm{C}$ layer, and the middle of the vacuum slab: $\mathrm{d} \approx 6.7 \AA$ (solid black line). The red/blue (dark/light gray) solid line shows the contribution from the areas enclosed within/between the $\mathrm{C}$ stripes, and the red/blue (dark/light gray) dashed line the corresponding contribution from the minority-spin states. The zero of energy corresponds to the Fermi level. The densities of states have been convoluted with a Gaussian of standard deviation $0.1 \mathrm{eV}$.

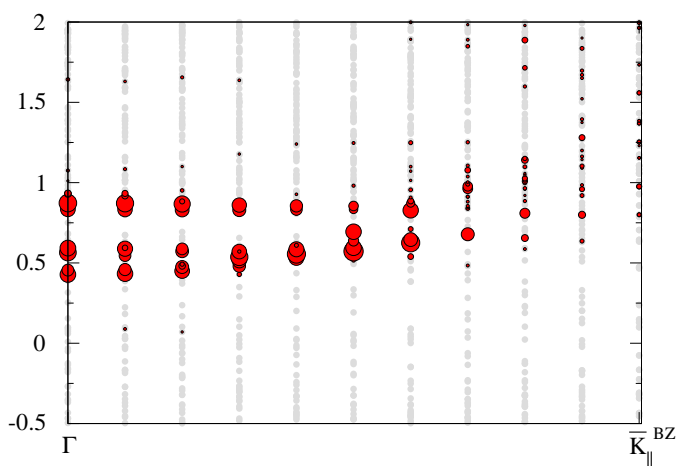

FIG. 4: (Color online) Calculated surface band structure of the minority-spin tunneling states with largest integrated probability density in the vacuum space beyond $\mathrm{d} \approx 2.3 \AA$. The dispersion is shown along the $\bar{\Gamma}-\bar{K}_{\|}^{B Z}$ line parallel to the $\mathrm{C}$ chains in the Brillouin zone of the $\mathrm{c}(3 \sqrt{2} \times \sqrt{2})$ surface; $\bar{K}_{\|}^{B Z}$ stands for the zone-edge $k$ point $\left(\frac{\pi}{2 a}, \frac{\pi}{2 a}\right)$. The eigenvalues are indicated with gray points, while the filled red (dark gray) circles indicate states that have a non-negligible charge density beyond $\mathrm{d} \approx 2.3 \AA$. The radii of the circles are proportional to the integrated charge density of the corresponding states between $\sim 2.3 \AA$ and $\sim 6.7 \AA$. The zero of energy corresponds to the Fermi level. 

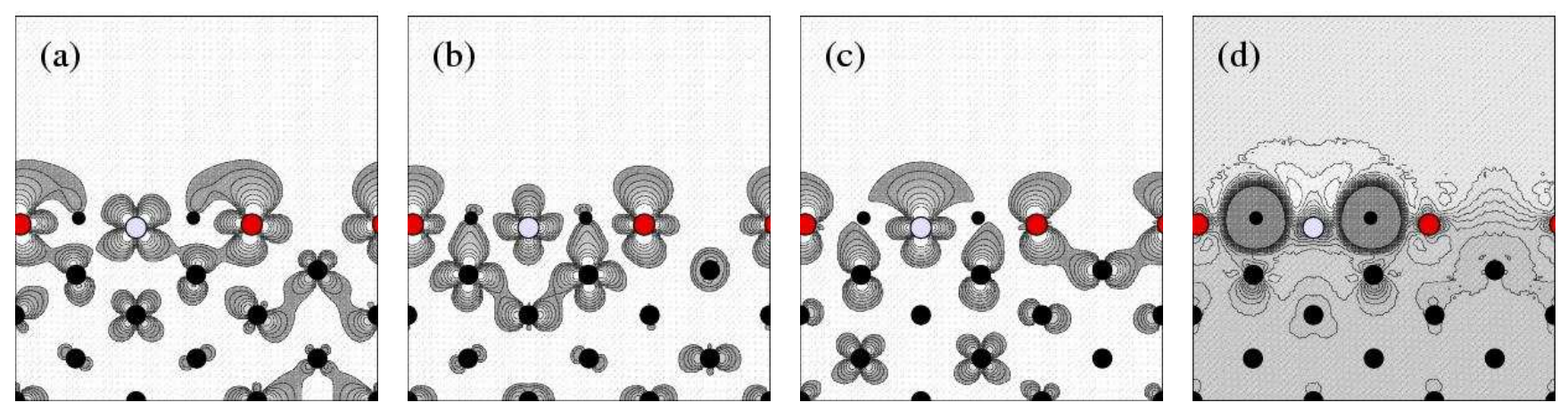

FIG. 5: (Color online) Charge density of the minority spin states at $\bar{\Gamma}$ with energy $0.43 \mathrm{eV}$ (a), $0.56 \mathrm{eV}(\mathrm{b})$ and $0.83 \mathrm{eV}(\mathrm{c})$. The contour plots are displayed in an atomic plane perpendicular to both the surface and the $\mathrm{C}$ chains. The $\mathrm{C}, \mathrm{Fe}_{1}$, and $\mathrm{Fe}_{2}$ atoms are indicated with small black disks, large red disks, and large light blue disks, respectively; other iron atoms are indicated with large black disks. A logarithmic scale common to the three plots has been used: the lowest contour corresponds to $10^{-4} \mathrm{e} / \mathrm{bohr}^{3}$ and the value increases by a factor of 1.85 from one countour to the next. In panel $(\mathrm{d})$ we display, in the same plane, the change in the electrostatic potential induced by the $\mathrm{C}$ atoms. The mean electrostatic potential is set to zero in the bulk, and the contours are separated by $0.15 \mathrm{eV}$. The potential increases from dark to light gray regions. Negative values are truncated below $-1.8 \mathrm{eV}$.

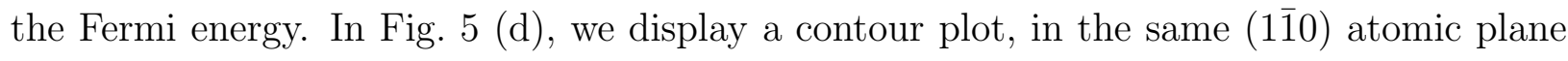
as in Figs. [ 5 (a,b,c), of the electrostatic potential change induced by the $\mathrm{C}$ atoms at the $\mathrm{Fe}$ $c(3 \sqrt{2} \times \sqrt{2})$ surface. The corrugation induced by the presence of the $\mathrm{C}$ atoms gives rise to a region in the vacuum, in front of the $\mathrm{Fe}_{2}$ stripes, in which the potential is globally repulsive with respect to the potential found, at the same distance from the surface, in front of the $\mathrm{Fe}_{1}$ atomic rows. Such a corrugation of the electrostatic potential tends thus to shift the Fe $d_{3 z^{2}-r^{2}}$ like surface states of the $\mathrm{Fe}_{2}$ atoms to higher energy with respect to those of the $\mathrm{Fe}_{1}$ atoms, leading to a localization of the resulting low/high energy surface states within/in between the $\mathrm{C}$ stripes.

In summary, we predict one-dimensional minority-spin empty surface states near the Fermi energy at the $\mathrm{C} / \mathrm{Fe}(001) \mathrm{c}(3 \sqrt{2} \times \sqrt{2})$ surface. These states derive from $d_{3 z^{2}-r^{2}}$-like surface states of the clean $\mathrm{Fe}(001)$ surface and are laterally confined within the self-assembled carbon chains. Simulation of the STM images indicates that the zigzag 
structure observed in STM experiments at positive biases corresponds to the zigzag chains of iron atoms enclosed within the $\mathrm{C}$ stripes, and that the STM signal is largely due to the one-dimensional Fe surface states. Furthermore, we predict that the one-dimensional minority-spin surface states gives rise to a significant feature at $\sim 0.6 \mathrm{eV}$ above the Fermi energy, which should be observable in (spin-resolved) STS experiments.

We thank J. Fujii, G. Panaccione, I. Vobornik and G. Rossi for having drawn our attention on this system and for many helpful discussions. We would like also to thank M. Altarelli, P. Messina, and N. Stojic for very useful discussions. One of the authors (G. T.) acknowledges the support by Agatino Trimarchi during the first phase of this project. The calculations in this work have been carried out using the PWscf package. 14

1 M. F. Crommie, C. P. Lutz, and D. M. Eigler, Science 262, 218 (1993).

2 See, e.g., Ph. Avouris and I. W. Lyo, Science 264, 942 (1994); S. Shiraki, H. Fujisawa, N. Nantoh, and M. Kawai, Phys. Rev. Lett. 92, 96102 (2004).

3 A. Biedermann, O. Genser, W. Hebenstreit, M. Schmid, J. Redinger, R. Podloucky, and P. Varga, Phys. Rev. Lett. 76, 4179 (1996).

4 N. Memmel, Surf. Sci. Rep. 32, 91 (1998).

5 See, e.g., E. E. Vdovin, A. Levin, A. Patane, L. Eaves, P. C. Main, Y. N. Khanin, Y. V. Dubrovskii, M. Henini, and G. Hill, Science 290, 122 (2000).

6 See, e.g., D. Fuster, M. U. Gonzalez, L. Gonzalez, Y. Gonzalez, T. Ben, A. Ponce, S. I. Molina, and J. Martinez-Pastor, Appl. Phys. Lett. 85, 1424 (2004); H. W. Yeom, S. Takeda, E. Rotenberg, I. Matsuda, K. Horikoshi, J. Schaefer, C. M. Lee, S. D. Kevan, T. Ohta, T. Nagao, and S. Hasegawa, Phys. Rev. Lett. 82, 4898 (1999).

7 J. Fujii, M. Galaktionov, L. Giovanelli, G. Panaccione, F. Bondino, I. Vobornik, and G. Rossi, Thin Solid Films 428, 30 (2003).

8 G. Panaccione, J. Fujii, I. Vobornik, G. Trimarchi, N. Binggeli, G. Goldoni, R. Larciprete, and G. Rossi, unpublished.

9 J. P. Perdew, K. Burke, and M. Ernzerhof, Phys. Rev. Lett. 77, 3865 (1996).

10 D. Vanderbilt, Phys. Rev. B 41, 7892 (1990). 
11 J. Tersoff and D. R. Hamann, Phys. Rev. Lett. 50, 1998 (1983); W. A. Hofer, A. S. Foster, and A. L. Shluger, Rev. Mod. Phys. 75, 1287 (2003).

12 J. A. Stroscio, D. T. Pierce, A. Davies, R. J. Celotta, and M. Weinert, Phys. Rev. Lett. 75, 2960 (1995).

13 M. M. J. Bischoff, C. Konvicka, A. J. Quinn, M. Schmid, J. Redinger, R. Podloucky, P. Varga, and H. van Kempen, Phys. Rev. Lett. 86, 2396 (2001).

14 S. Baroni, A. dal Corso, S. de Gironcoli, and P. Giannozzi, URL http://www.pwscf.org/ 